Communications in Physics, Vol. 25, No. 4 (2015), pp. 359-365

DOI:10.15625/0868-3166/25/4/0

\title{
STRONG ELECTRON CORRELATION IN CU-DOPED CAO NANOCOLLOID
}

\author{
NGUYEN THUY TRANG \\ Faculty of Physics, Hanoi University of Science, Vietnam National University, Hanoi \\ HOANG NAM NHAT \\ Faculty of Engineering Physics and Nano Technology, University of Engineering and Technology, \\ Vietnam National University, Hanoi \\ E-mail:nhathn@vnu.edu.vn
}

Received 29 November 2015

Accepted for publication 24 December 2015

\begin{abstract}
Theoretical prediction of magnetism induced by defects or doping in non-metallic colloids has gained a renewed interests recently. In this work, we investigated the possible appearance of magnetism in $\mathrm{Cu}$ doped $\mathrm{CaO}$ nanocolloids activated by SPAN-80 in the framework of density functional theory (DFT). Despite of strong antiferromagnetic superexchange interaction between $\mathrm{Cu}^{2+}$ ions, the local magnetic moment of $\mathrm{Cu}$ may arise due to attachment of colloidal agent onto the surface of $\mathrm{CaO}$ nanocluster. The ferromagnetism attributes to the degeneration of $\mathrm{Cu} 3 \mathrm{~d}$ orbitals in $\mathrm{CaO}$ crystal fields, the aspects of electron correlation and quantum spin fluctuation.
\end{abstract}

Keywords: DFT, band-gap, CaO, calcium-oxide, mBJ, PBE, functional.

\section{INTRODUCTION}

Owing to the wide scope of application from optoelectronics to biology the rock salt compounds continuously attract the growing interests of scientists worldwide. A defect induced photoemission in $\mathrm{CaO}$ was a subject of many researches [1] and this popular rock salt proposed itself as a brilliant candidate for application in laser windows, IR domes, IR optical fiber... Recently, there has been a renewed interest in $\mathrm{CaO}$ due to a possibility of magnetism in doped (non-metallic) $\mathrm{CaO}$ and defect-induced metallic $\mathrm{CaO}$ compounds [2-5]. On the basis of tight-binding-like model, I. S. Elfimov et al. [2] showed that the cation vacancies in the divalent monoxides with rock salt structure as well as in any other compounds with octahedral coordination might possess the local magnetic moments if the on-site Coulomb repulsion $\mathrm{U}$ was taken into account. Their ab initio calculation for $\mathrm{CaO}$ with $3.125 \% \mathrm{Ca}$ vacancies revealed some local magnetic moments at the vacancy sites and the obtained numerical results were in good agreement with the theoretical expectation. Their calculation demonstrated the importance of inclusion of strong electron correlation when modeling the divalent monoxides. They also showed that $\mathrm{CaO}$ with vacancies exhibited a half-metallic ferromagnetic ground state. The experimental verification was obtained from the 
magnetization measurements taken at room temperature for $\mathrm{CaO}$ nanopowder [3]. Another possible mechanism to induce the local magnetic moments in $\mathrm{CaO}$ without doping of transition metals was proposed by $\mathrm{K}$. Kenmochi et al. [4] was to dope $\mathrm{CaO}$ with non-metallic elements such as $\mathrm{C}$, $\mathrm{N}$ and $\mathrm{B}$. The calculations in the framework of Korringa-Kohn-Rostocker method predicted that these materials should exhibit the room temperature ferromagnetism with local moments originating from $2 p$ orbitals of dopants. The effects of the Coulomb repulsion $U$ within $2 p$ orbitals were investigated in more details by V. Pardo and W. E. Pickett [5]. According to their results, the inclusion of $U$ had an effect of strengthening the localization of magnetic moments and reducing the possible magnetic interaction.

In this work, we show that the local magnetic moments may be obtained in $\mathrm{Cu}$-doped $\mathrm{CaO}$ nanocolloids activated by using SPAN-80 as colloidal agent. It is well-known that the high temperature superconducting cuprates possess the antiferromagnetic Mott-insulating ground state due to the superexchange interaction between the strongly correlated spin $1 / 2 \mathrm{Cu}^{2+}$ cations situating in the octahedral crystal field of surrounding oxygen atoms. It is thus reasonable to expect that the octahedral field substitution of $\mathrm{Cu}^{2+}$ for $\mathrm{Ca}^{2+}$ may inject some amounts of local magnetic moments while introducing no extra electrons or holes. However, the question is whether or not $\mathrm{Cu}^{2+}$ remains spin-polarized in the crystal field of rock salt as expected and if polarized then which magnetic interaction, ferromagnetic or antiferromagnetic, is preferable. In the framework of density functional theory (DFT), we aim at clarifying this problem

\section{CALCULATIONS DETAILS}

Both conventional orbital-independent exchange-correlation functionals i.e. generalized gradient approximation (GGA) type and orbital-dependent correlation functionals, i.e. GGA+U were used. The GGA functional is PBE of which the exchange part was formulated by Beck and the correlation part was by Perdew-Wang [6]. The GGA+U is a kind of a mixture between density functional theory method and Hubbard model theory in which the semilocal formulation of exchange-correlation of a considered orbital is substituted with the Hubbard one to give a better description of strongly-correlated system [7]. To formulate the orbitals for system considered, the numerical atomic-like basis set and plane wave basis set with norm-conserving pseudopotential (PWPP) were used in combination with GGA and GGA+U methods respectively. The numerical atomic-like basis set is composed of a double-numeric quality basis set, i.e. 2 atomic orbitals for each 1 occupied in free atom plus polarization functions with angular momentum one higher than that of the highest occupied orbital in free atom (DNP basis set). The sets of DNP/GGA and $\mathrm{PWPP} / \mathrm{GGA}+\mathrm{U}$ methods were provided by $\mathrm{Dmol}^{3}$ and CASTEP codes respectively $[8,9]$.

\section{RESULTS AND DISCUSSIONS}

First, we examined the periodic boundary condition (PBC) by using the DNP/GGA method how two $\mathrm{Cu}$ atoms can be inserted into $\mathrm{Ca}$ sites using a large suppercell $2 \times 2 \times 2$ (Fig. 1(a)), which particularly corresponds to the impurity level of $6.25 \%$. Three doping configurations with highest symmetry were considered: two $\mathrm{Cu}$ atoms aligned along (111), (101) and (001) directions. At each configuration, the initial ordering of spins was set to ferromagnetic (FM) and antiferromagnetic (AFM) respectively. The comparison of obtained total ground state energies revealed that the (001) configuration was the most preferable one which had $\mathrm{E}_{\text {total }}(001) 7 \mathrm{eV}$ lower than that 
of the other two. Table 1 summarizes the total energy $\mathrm{E}_{\text {total }}$, concentrated charge $\mathrm{Q}_{C u}$ and spin $\mu_{C u}$ from Mulliken's electron population analysis, lattice constant and effective exchange constant $\mathrm{J}_{\text {eff }}$ calculated using Heisenberg model. According to the data presented, while (111) configuration preferred the AFM ordering state, the (101) preferred the FM one. However the obtained $\mathrm{Cu}$ local moments were very small, i.e. $\mu_{C u} \approx 0.2 \%$ of the moment of $\mathrm{Cu}^{2+}$. For the $(001)$ configuration with both FM and AFM initial magnetic orderings, the electron density converge to the nonmagnetic state with zeroed $\mathrm{Cu}$ local moments.

Table 1. Summarization of total energy $\mathrm{E}_{t o t a l}$, concentrate atomic charge on $\mathrm{Cu} \mathrm{Q}_{C u}$, local magnetic moment of $\mathrm{Cu} \mu_{C u}$, effective exchange interaction $\mathrm{J}_{e f f}$ and magnetic ground state for $\mathrm{Ca}_{0.9375} \mathrm{Cu}_{0.0625} \mathrm{O}$

\begin{tabular}{|c|c|c|c|c|c|c|}
\hline & \multicolumn{2}{|c|}{ (111) } & \multicolumn{2}{|c|}{ (101) } & \multicolumn{2}{|c|}{$(001)$} \\
\hline $\begin{array}{c}\text { Initial } \\
\text { magnetic order }\end{array}$ & FM & AFM & FM & AFM & FM & AFM \\
\hline $\begin{array}{c}E_{\text {total }} \\
(\mathrm{Ha} / \text { cell })\end{array}$ & -26748.586702 & -26748.586706 & -26748.586707 & -26748.586702 & -26748.609028 & -26748.609028 \\
\hline $\mathrm{Q}_{C u}(|\mathrm{e}|)$ & 0.539 & 0.539 & 0.539 & 0.539 & 0.464 & 0.464 \\
\hline$\left|\mu_{C u}\right|\left(\mu_{B}\right)$ & 0.002 & 0.002 & 0.002 & 0.002 & 0 & 0 \\
\hline$J_{e f f}(\mathrm{meV})$ & \multicolumn{2}{|c|}{-0.055} & \multicolumn{2}{|c|}{0.070} & \multicolumn{2}{|c|}{0} \\
\hline Ground state & \multicolumn{2}{|c|}{ AFM } & \multicolumn{2}{|c|}{ FM } & \multicolumn{2}{|c|}{ Paramagnetic or spin-glass? } \\
\hline
\end{tabular}

Investigating the response of the $\mathrm{PBC}$ doped structure putting within the cavity of additional Coulomb force-field introduced by solvent by using the Conductor-like Screening Model (COSMO) for the surrounding media with dielectric constants from 2 (common oils) to 80 (water) we observed a slight enhancement of local moments (less then $0.1 \%$ ) of doped sites when the dielectric constant of medium decreased. This effect may be assigned to the strengthening of Coulomb repulsion between occupied and unoccupied states due to the occurrence of additional Coulomb field at the surface of the PBC structure. However, the prediction of COSMO for oils (including SPAN-80) was known to be twice as large as the prediction by ab initio using the direct attachment of colloidal agent onto the nanocluster surface.

The strong reduction of $\mathrm{Cu}$ local moment in the $\mathrm{PBC}$ structure can be assigned to three possible reasons: (1) the strong antiferromagnetic quantum spin fluctuation (2) the intrinsic splitting of $3 \mathrm{~d} \mathrm{Cu}$ orbital in crystal field of $\mathrm{CaO}$ and (3) the inadequate treatment on strong correlation aspects, i.e. insufficient on-site Coulomb repulsion $\mathrm{U}$. Because the $\mathrm{Cu}$ local moment are the same for both FM and AFM magnetic ordering states in (111) and (101) configurations, the quantum spin fluctuation should be possible. It is easy to see that $\mathrm{Cu}$ atoms occupy sites so that $\mathrm{Cu}-\mathrm{O}$ chains were formed in two and three dimensions The situation is quite similar to the one as in $\mathrm{Sr}_{2} \mathrm{CuO}_{3}$ and $\mathrm{Ca}_{2} \mathrm{CuO}_{3}$ where the $\mathrm{CuO}$ layers are separated from each other by two $(\mathrm{Sr} / \mathrm{Ca}) \mathrm{O}$ layers. Consequently, the interlayer coupling was small in comparison with the intrachain interaction [10]. To be specific, the value is about 10 times smaller than the effective exchange $\mathrm{J}_{\text {eff }} \approx 1 \mathrm{meV}$ as obtained for $\mathrm{Ca}_{0.9375} \mathrm{Cu}_{0.0625} \mathrm{O}$ in (111) and (101) configuration. Therefore, it is possible that the small local magnetic moment $\mu_{C u}=0.05 \mu_{B}$ was due to the strong quantum spin fluctuation which suppresses $95 \%$ magnetic moment of $\mathrm{Cu}$ site $[11,12]$. 
In order to figure out the roles of crystal field splitting and on-site Coulomb repulsion we note that the calculation on basis of tight binding-like model for a cluster of oxygen octahedron by I. S. Elfimov et al. [2] indicated that whether or not the vacancy will possess the spin-polarized ground state depends on the crystal field splitting of orbitals. It was the nature of hole orbitals which were formed mainly by $2 p$ orbitals of oxygen that they were degenerate, therefore in the presence of strong Coulomb repulsion the singlet states should be lifted to higher energy in comparison with the triplets. It is also well-known that the on-site Coulomb repulsion prefers the single occupancy of spin-polarized states while the crystal splitting induces the double occupancy of spin-unpolarized state. According to this, when $\mathrm{Cu}$ was substituted for $\mathrm{Ca}$ in its octahedral field of surrounding oxygen, five $\mathrm{Cu} 3 \mathrm{~d}$ orbitals are three-fold degenerate orbitals $t_{2 g}$ (which are full-filled) and two-fold degenerate orbitals $e_{g}$ (which are partial-filled). With a lack or inadequate treatment of electron repulsion a ground state of the doped compound may appear as spin glass or nearly spin glass, with $\mu_{C u}=0$ or nearly zero.

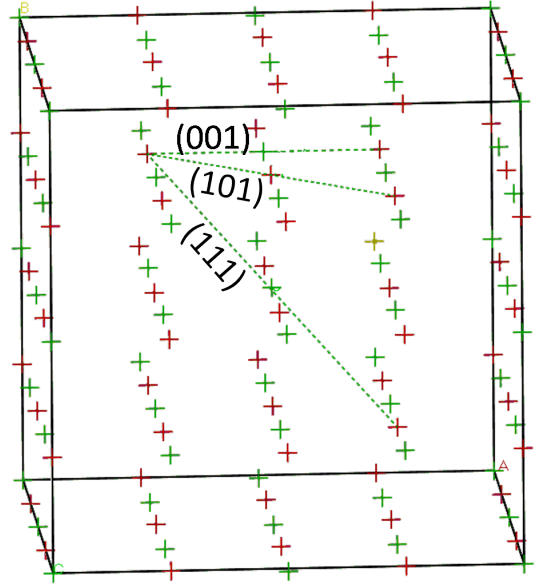

(a)

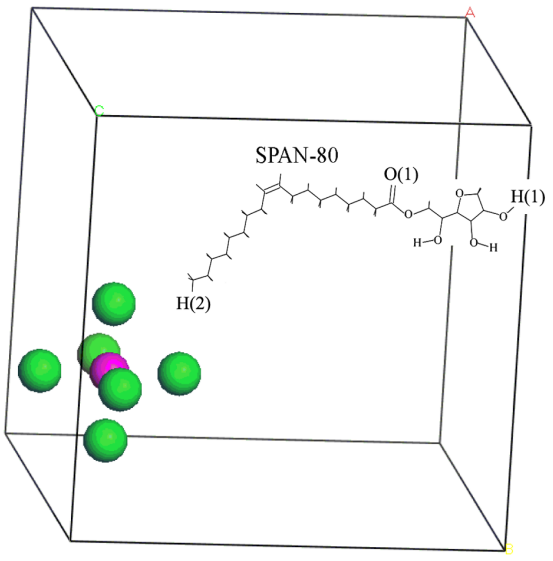

(b)

Fig. 1. (a) $\mathrm{CaO}$ supercell $2 \times 2 \times 2$ modeling a doping level $6.25 \%$. The green dash line indicate (001), (101) and (111) direction. (b) A supercell of octahedral nanocluster $\mathrm{CuO}_{6}$ embedded in vacuum; the inset shows the SPAN-80 molecule with its $\mathrm{H}(1)$ center.

Then we utilized the model cluster as given in Fig. 1(b). The approximation of choice was the PWPP/GGA+U as described above. First we tested the model with $U=0,2.5,3,4,5,6 \mathrm{eV}$ applied on the $\mathrm{Cu} 3 \mathrm{~d}$ orbitals. The results showed that the total magnetic moment was around the nominal value of $1 \mu_{B}$ and nearly $50 \%$ was concentrated on $\mathrm{Cu}$ for all values of $\mathrm{U}$ (Fig. 2(a)). The local magnetic moment even appeared for $U=0$ which indicated that the plane wave basis set can reproduce the electron correlation in octahedral coordination better than the numerical atomic-like basis set. Fig3(a) and (b) show the partial density of spin states (DOS) calculated with various values of $U$. The addition of the on-site Coulomb term seemed to make the $\mathrm{Cu} 3 \mathrm{~d}$ spins more tightly bound to the core, in other words, they were more localized. This in turn reduced the exchange constants which were observed to usually be larger than the measured ones. 


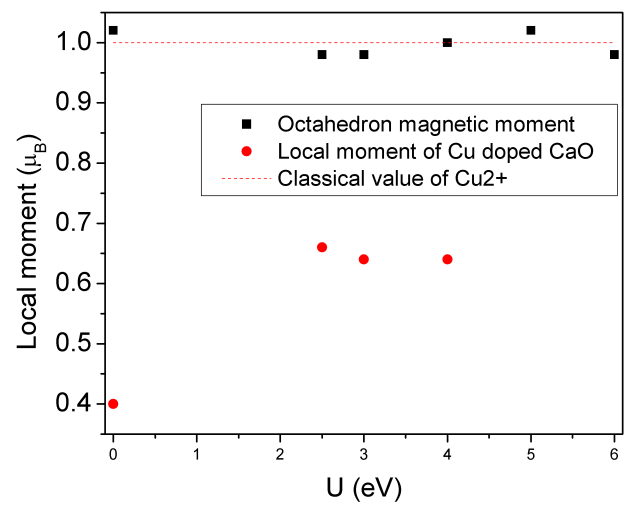

(a)

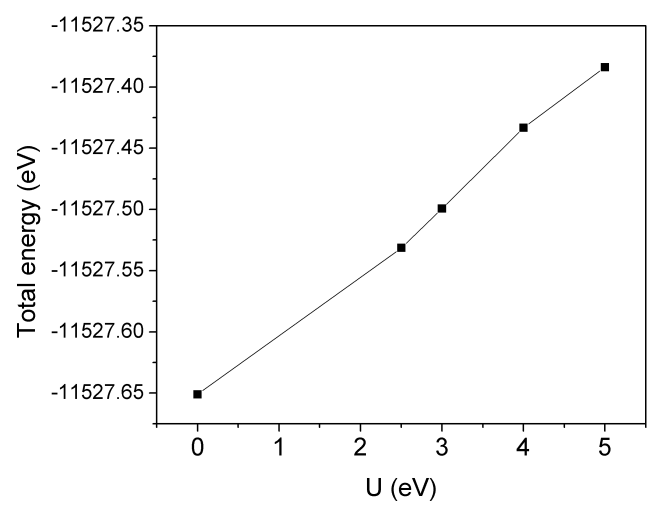

(b)

Fig. 2. (a) Plot of the local magnetic moment according to $\mathrm{U}$ for $\mathrm{CuO}_{6}$ nanocluster (black close squares) and $\mathrm{Cu}$ doped $\mathrm{CaO}$ (red close round). The red dash line denotes the nominal magnetic moment of $\mathrm{Cu}$. (b) The dependence of total energy of AFM state of $\mathrm{Cu}$ doped $\mathrm{CaO}$ on $\mathrm{U}$ value

Applying this methodology on the $\mathrm{PBC}$ structure of $\mathrm{Cu}$ doped $\mathrm{CaO}$ with doping concentration $25 \%$ using a supercell of the size $1 \times 1 \times 2$ and initial spin ordering set to AFM, we found a smaller local moment of maximum $0.66 \mu_{B}$ (corresponding to $\mathrm{U}=2.5,3,4 \mathrm{eV}$ ) The value reduced to $0.48 \mu_{B}$ for $\mathrm{U}=0 \mathrm{eV}$ and to $0.38 \mu_{B}$ for large $\mathrm{U}=5 \mathrm{eV}$. Fig 2 expresses the dependence of local moment and energy difference between AFM and nonmagnetic spin ordering states $\Delta \mathrm{E}$ on U. Fig. 3(c) and (d) show the partial density of spin states (DOS) calculated for $\mathrm{U}=0$ and $4 \mathrm{eV}$. It appeared reasonably that almost all spin-polarization was originated from $\mathrm{O} 2 \mathrm{p}$ and $\mathrm{Cu} 3 \mathrm{~d}$ states. As expected, the reduction of $\Delta \mathrm{E}$ when $\mathrm{U}$ increased seemed to correspond with the reduction of exchange amplitude due to the localization of spins.

With the settings given we studied the COSMO effect on final local moment for the surrounding media having the dielectric constant from 2 to 80. Again we have observed a slight enhancement of measure not greater than $0.5 \%$. This result argued for the fact that on PBC structure of 25\% doping the COSMO effect only limited response. However, the increase was observably larger if one applied COSMO on doped nanocluster with $25 \% \mathrm{Cu}$ content. We recorded the increase of almost $27 \%$, that is the maximum local moment over $\mathrm{Cu}$ site achieved $0.84 \mu_{B}$ But as noted above, the COSMO results were usually overestimated by the ratio approximately equal $100 \%$ for oils (thus for SPAN-80 in our case of interest). Its error is small only for the polar solvents like water (with dielectric constant near 80). For the purpose of more exact estimation of the effect of solvent binding on surface of doped cluster we built $\mathrm{H}(1)$ center of one molecule SPAN-80 (Fig.1 (b)) onto the $\mathrm{O}$ center of doped $\mathrm{CaO}$ nanocluster. The previous study from our group [13] showed that the binding through $\mathrm{H}(2)$ and $\mathrm{O}$ centers of SPAN-80 were not preferred. Such the bindings resulted in the higher ground state energy $(i 1 \mathrm{eV})$ of the binary nanoparticlecolloid system in comparison with the case of binding through $\mathrm{H}(1)$. The optimized geometry was achieved at the O...H(1) distance of $1.7 \AA$ This distance is short enough to replicate the COSMO effect on the $\mathrm{Cu}$ site directly bound to the given $\mathrm{O}$ center. As expected, the local moment reduced to smaller value of $0.76 \mu_{B}$, that is the binding induced only $\approx 14 \%$ increase of local moment. 

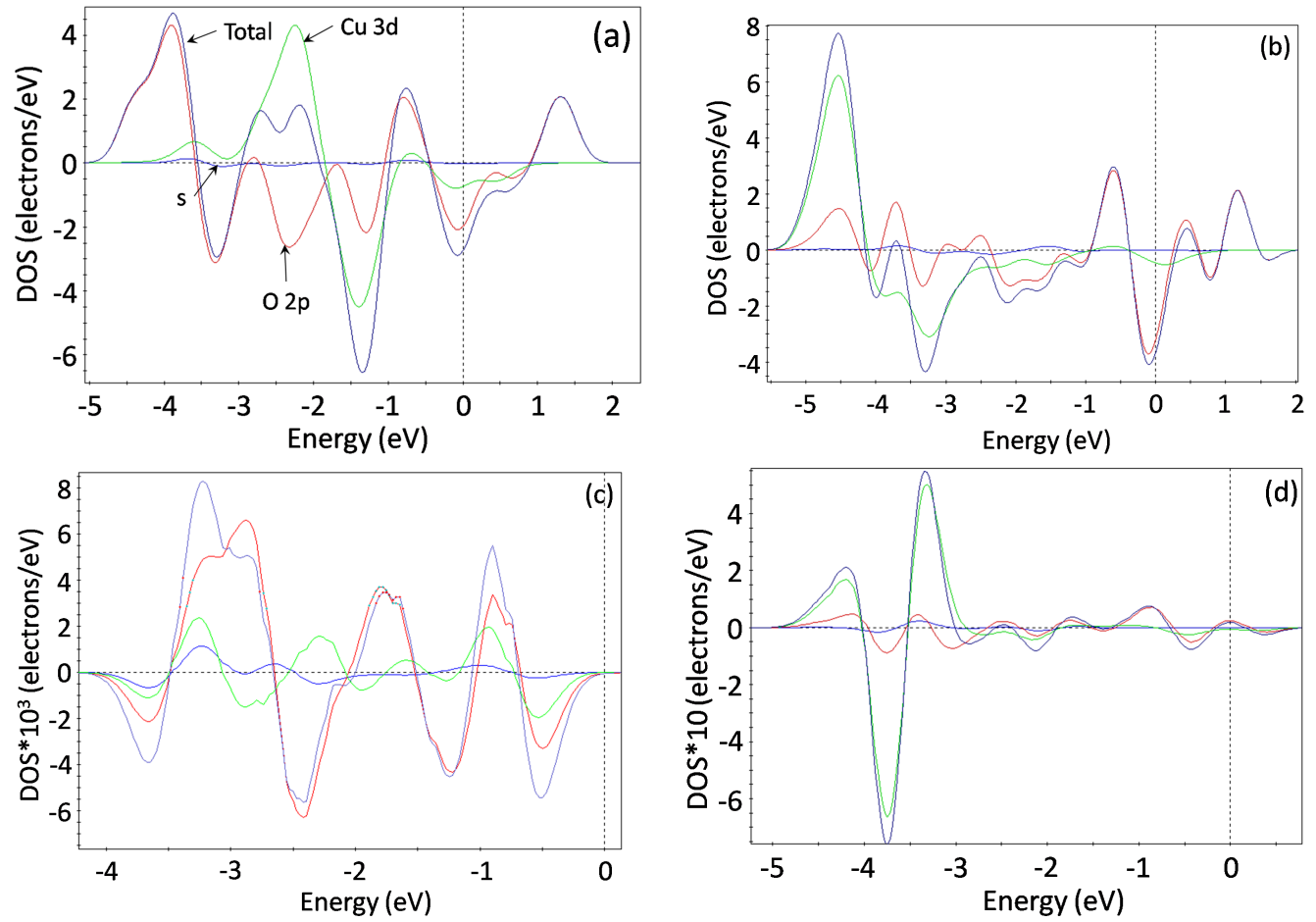

Fig. 3. The partial density of spin states (DOS) calculated by using PWPP/GGA+U method for the octahedral $\mathrm{CuO}_{6}$ nanocluster (a) $\mathrm{U}=0 \mathrm{eV}$; (b) $\mathrm{U}=4 \mathrm{eV}$. The same for $\mathrm{Cu}$ doped $\mathrm{CaO}(\mathrm{c}) \mathrm{U}=0 \mathrm{eV}$; and (d) $\mathrm{U}=4 \mathrm{eV}$.

However, we should note here the difference between modeling the additional Coulomb force field (ACFF) by COSMO and by direct surface binding of surfactant. While COSMO estimates ACFF by putting solute inside a cavity of surfactant so the charge polarization (like in a capacitor) is built-up onto two sides of a vacuum layer between solute and surfactant thus introducing the ACFF on solute, the modeling of ACFF by direct binding of surfactant onto surface of solute estimates ACFF by the strength of exchange interaction between bonding atoms. In our case, more electrons were depleted from $\mathrm{H}(1)$ center of SPAN-80 and pumped onto the $\mathrm{O} 2 \mathrm{p}$ orbital, raising its overall occupation level, which in turn induced a stronger Coulomb repulsion between occupied and unoccupied states.

\section{CONCLUSION}

The possible appearance of ferromagnetism in non-magnetic insulating materials was always exciting phenomenon. Here we examined such possibility in $\mathrm{Cu}$ doped $\mathrm{CaO}$ nanocolloid within the framework of DFT methods. We showed that the inclusion of strong electron correlation was important to discover the electron localization effect The obtained results argued for a large local magnetic moment upon $\mathrm{Cu}$ doping sites and possible appearance of ferromagnetism in doped nanoclusters where the ferromagnetic penetration depth is comparable with the cluster size. We leave this for future consideration. 


\section{ACKNOWLEDGEMENT}

This research is funded by Vietnam National Foundation for Science and Technology Development (NAFOSTED) under grant number 103.02-2014.17. One of the authors, Nguyen Thuy Trang, would like to thank TRIG A Project of Hanoi University of Science, Vietnam National University, Hanoi for supporting.

\section{REFERENCES}

[1] B. Henderson, S.E. Stokowski and T.C. Ensign, Phys. Rev. 183(3) (1969) 826-831; S. C. Ke, Phys. Rev. B 62(7) (2000) 4194-4195; H.C. Hafner, N.J. Kreidl, and R.A. Weidel, J. Am. Ceram. Soc. 41 (1958) 315-323.

[2] I. S. Elfimov, S. Yunoki and G. A. Sawatzky, Phys. Rev. Lett. 89 (2002) 216403.

[3] Daqiang Gao, Jinyun Li, Zhuoxin Li, Zhaohui Zhang, Jing Zhang, Huigang Shi, and Desheng Xue, J. Phys. Chem. C 114 (2010) 11703-11707.

[4] K. Kenmochi, M. Seike, K. Sato, A. Yanase and H. Katayama-Yoshida, Japanese Journal of Applied Physics 43(7A) L934-L936 (2004).

[5] V. Pardo and W. E. Pickett, Phys. Rev. B 78, 134427 (2008).

[6] J. P. Perdew, K. Burke, M. Ernzerhof, Phys. Rev. Lett. 77 (1996) 3865 .

[7] V. I. Anisimov, J. Zaanen, and O. K. Andersen, Phys. Rev. B 44 (1991) 943-954.

[8] B. Delley, J. Chem. Phys. 92 (1990) 508.

[9] J. S. Lin, A. Qteish, M. C. Payne, V. Heine, Phys. Rev. B 47 (1993) 4174-4180; M. H. Lee, PhD Thesis, Cambridge University (1996).

[10] T. Ami, M.K. Crawford, R. L. Harlow, Z. R. Wang, D. C. Johnston, Q. Huang, and R.W. Erwin, Phys. Rev. B 51 (1995) 5994.

[11] Coen de Graaf, Francesc Illas, Phys. Rev. B 63 (2001) 014404; H. Rosner, H. Eschrig, R. Hayn, S.-L. Drechsler, J. Malek, Phys. Rev. B 56 (1997) 3402 .

[12] K. M. Kojima, Y. Fudamoto, M. Larkin, G. M. Luke, J. Merrin, B. Nachumi, Y. J. Uemura, N. Motoyama, H. Eisaki, S. Uchida, K. Yamada, Y. Endoh, S. Hosoya, B. J. Sternlieb, and G. Shirane, Phys. Rev. Lett. 78 (1997) 1787

[13] Thu Huong Ngo, Hong Van Bui, Van Ben Pham, Thi Hong Tran, An Bang Ngac, Nam Nhat Hoang, J. of Lumin. 132 (2012) 2135 